\title{
AVALIAÇÃO DA EFICÁCIA DO ARTESUNATO ASSOCIADO À TETRACICLINA NA TERAPÊUTICA DA MALÁRIA FALCIPARUM
}

\author{
Juan Miguel Villalobos Salcedo, Luís Marcelo Aranha Camargo, \\ Maria de Fátima Vital Braga, Patrícia Soares de Maria \\ e Vanize de Oliveira Macêdo
}

\begin{abstract}
Realizou-se um ensaio clinico, randomizado e controlado, comparando o artesunato com o quinino e a mefloquina, em casos de malária não grave. Foram tratados 42 pacientes em regime de internação e o seguimento durou 28 dias. Realizouse exame de gota espessa cada 12 boras até sua negativação, hemograma e bioquímica sanguínea, pré e pós-tratamento. A média da parasitemia inicial foi 42.568 parasitas $/ \mathrm{ml}$. Vinte e seis pacientes foram acompanhados durante 28 dias e 16 durante menos de 28 dias. Um paciente de cada grupo apresentou $R$ I tardia e um paciente do grupo do quinino apresentou $R$ III. As porcentagens de cura foram 88,8\%, 85, 7\% e 81,8\% para o artesunato, a mefloquina e o quinino, respectivamente, sem mostrar diferença significativa. O tempo de desaparecimento da febre não mostrou diferença significativa entre os grupos. O grupo do artesunato teve um tempo menor de clareamento da parasitemia $(37,33 \pm 11,52$ boras $)$ quando comparado com o quinino $(65,25 \pm 17,44$ horas), sendo estatisticamente significativa ( $p=0,0016)$. O grupo da mefloquina (58,9 $\pm 16,68$ horas) não mostrou diferença com os outros grupos. Não se apresentaram efeitos adversos importantes em nenhum dos esquemas usados, sendo bem tolerados pelos pacientes.
\end{abstract}

Palavras-chaves: Malária. Artemisinina. Artesunato. Ensaio clínico.

A malária é a mais importante doença parasitária dos trópicos com uma população de risco que corresponde a $32 \%$ da população mundial. Estima-se em 300 milhões o número de pessoas infectadas, 120 milhões de casos novos e cerca de um a dois milhões de óbitos por ano33.

A incidência na última década aumentou nas Américas e na Ásia, sendo que 75\% do número de casos novos (excluindo Affrica), concentramse em nove países: Índia, Brasil, Afeganistão, Sri-Lanka, Tailândia, Indonésia, Vietnã, Camboja e China. Nas Américas, em 1974 relataram-se 269.000 casos, aumentando para $1.057 .000 \mathrm{em}$ 1991, dos quais 560.000 foram comunicados pelo Brasil (53\%)33.

\footnotetext{
Núcleo de Medicina Tropical e Nutrição, Universidade de Brasília, DF. Centro de Medicina Tropical de Rondônia, Porto Velho, RO. Departamento de Parasitologia, Instituto de Ciências Biomédicas, Universidade de São Paulo, São Paulo, SP. Este trabalho foi realizado com apoio financeiro do Núcleo de Medicina Tropical e o Conselho Nacional de Pesquisa (CNPq).

Endereço para correspondência: Dr. Juan Miguel Villalobos Salcedo. Divisão de Pesquisa, CEMETRON. BR 364, km 35. Fax:(069) 221-3265.

Recebido para publicação em 03/04/95.
}

O Brasil representa um país único, em termos de diversidade de situações epidemiológicas. Noventa e nove por cento dos registros provêm da região Amazônica e dentro desta, os Estados de Pará, Rondônia e Mato Grosso, detêm mais de $80 \%$ destes. Rondônia relatou mais de 134.000 casos novos em 1995 (aproximadamente $20 \%$ do total), sendo que mais da metade é procedente de dez municípios, com incidências de até 750/1.000 habitantes e com fórmula parasitária que favorece a malária vivax $(60 \%) 20$.

A resistência às drogas pode ser considerada uma ameaça ao controle efetivo desta doença33. Em referência à resistência do Plasmodium falciparum, esta já foi amplamente documentada com relação a muitas drogas em diferentes países do mundo9 102335 . Considerando esta situação, a Organização Mundial da Saúde (OMS) determinou prioritário o desenvolvimento de esquizonticidas sanguíneos de ação rápida derivados da artemisinina para o tratamento da malária grave e/ou complicada e para o controle das cepas multirresistentes de P. falciparum.

A medicina tradicional chinesa usou por mais de 2.000 anos, uma planta conhecida como Artemisia annua L, com o propósito de "controlar e reduzir as febres"17. Em inícios dos anos 70, 
Salcedo JMV, Camargo LMA, Braga MFV, Maria PS, Macêdo VO. Avaliação da eficácia do artesunato associado à tetraciclina na terapêutica da malária falciparum. Revista da Sociedade Brasileira de Medicina Tropical 30:215-222, mai-jun, 1997.

os cientistas chineses extraíram o princípio ativo, chamado de qinghaosu ou artemisinina. Esta é uma sesquiterpena-lactona com uma ligação peróxido pouco usual na sua estrutura que determina uma importante atividade antimalárica, inclusive sobre isolados de $P$. falciparum multirresistentes.

A diidro-artemisinina, o meio-éster do ácido succínico, forma o sal sódico conhecido como artesunato sódico, o qual tem demonstrado efetividade em estudos clínicos realizados em diferentes países 42534 . No entanto, a artemisinina e seus derivados têm demonstrado um alto índice de recrudescência ${ }^{1} 13$, podendo variar de 10 até 50\%. Esta ampla diferença, parece ter relação com as doses utilizadas e o tempo de tratamento 22

O tratamento combinado com várias drogas, pretende retardar o aparecimento da multirresistência22. Com este conceito e o objetivo de aumentar a porcentagem de cura obtida com a artemisinina, tem-se testado várias combinações com outros antimaláricos11 131821. A combinação artemisinina e sulfadoxinapirimetamina 21 , não é recomendada pelo seu alto percentual de recrudescência $(44,8 \%)$, enquanto outras, como artemisinina associada a mefloquina 19 ou artemisinina associada a tetraciclina 711 , melhoram a porcentagem de cura e apresentam sinergismo in vitro 27.

O uso da tetraciclina no tratamento da malária8 tem sido proposto desde 1972 e seu emprego em associação com o quinino, é amplamente difundido no sudeste asiático e na região amazônica.

Este trabalho avaliou a eficácia do artesunato, num estudo clínico comparativo com o quinino e a mefloquina na terapêutica da malária falciparum não grave, em Porto Velho, Rondônia.

\section{MATERIAL E MÉTODOS}

Localidade do estudo. O estudo desenvolveuse no Centro de Medicina Tropical de Rondônia (CEMETRON), localizado no município de Porto Velho, capital do Estado de Rondônia. É um hospital que serve como centro de referência em medicina tropical para o Estado e conta com uma Divisão de Pesquisa, que funciona em forma de convênio entre a Secretaria Estadual de Saúde, o Instituto de Ciências Biomédicas da Universidade de São Paulo (USP) e o Instituto Pasteur da França.
O Estado de Rondônia tem uma superfície de $243.044 \mathrm{~km}^{2}$ e está situado na Amazônia Ocidental, ao sul da Região Norte. Cerca de 94\% da sua área, encontram-se acima dos 100 metros e abaixo dos $600 \mathrm{~m}$, em relação ao nível do mar. O clima é equatorial úmido, com temperaturas que variam entre $18{ }^{\circ} \mathrm{C}$ a $40{ }^{\circ} \mathrm{C}$. A umidade relativa do ar varia entre $73 \%$ e $88 \%$ e os índices pluviométricos anuais variam de $1.500 \mathrm{~mm}$ (verão: novembro a março) a $80 \mathrm{~mm}$ (inverno: maio a agosto).

Estrutura do estudo. O ensaio clínico foi comparativo, randomizado e controlado, dividindo os pacientes em cinco grupos:

Grupo 1: artesunato (vo) associado a tetraciclina (vo).

Grupo 2: artesunato (ev) associado a tetraciclina (vo).

Grupo 3: mefloquina (vo) sem associação.

Grupo 4: quinino (ev) associado a tetraciclina (vo).

Grupo 5: quinino (vo) associado a tetraciclina (vo).

O ensaio foi desenvolvido em duas fases: uma de internação de 4 dias e uma ambulatorial de 24 dias, completando-se assim 28 dias de seguimento.

População do estudo. Foram selecionados quarenta e dois pacientes que procuraram o CEMETRON, com diagnóstico parasitológico realizado pelos técnicos da Fundação Nacional de Saúde e com parasitemia quantificada em duas cruzes de malária falciparum, com residência fixa em Porto Velho e quadro clínico de malária não grave. Foram excluídas as gestantes e os pacientes que usaram drogas antimaláricas nos últimos 15 dias.

Diagnóstico parasitológico. O diagnóstico parasitológico realizou-se através do exame de gota espessa e esfregaço corados pelo Giemsa, segundo a técnica de Walker e, examinados em microscópio de luz. O paciente com duas cruzes de parasitemia foi submetido a novo exame para quantificação desta. A contagem parasitária foi realizada por 200 células brancas e feito o cálculo com base na constante de 5.000 leucócitos $/ \mathrm{ml}$. Considerou-se negativa quando o exame em objetiva de imersão não revelou parasitas em 200 campos 32.

Administração da droga. O quinino (ev) foi administrado na dose de $10 \mathrm{mg} / \mathrm{kg} / \mathrm{dose}$, diluído em solução glicosada 5\% e infundido 
Salcedo JMV, Camargo LMA, Braga MFV, Maria PS, Macêdo VO. Avaliação da eficácia do artesunato associado à tetraciclina na terapêutica da malária falciparum. Revista da Sociedade Brasileira de Medicina Tropical 30:215-222, mai-jun, 1997.

em quatro horas, a cada oito horas, durante três dias. O quinino (vo) na dose de $10 \mathrm{mg} / \mathrm{kg} /$ dose a cada oito horas durante três dias. A dose do artesunato (ev) foi de $1,5 \mathrm{mg} / \mathrm{kg}$ na hora $0,4,24$ e 48 . O artesunato (vo) administrouse na dose de $100 \mathrm{mg}$ iniciais, seguida de $50 \mathrm{mg}$ a cada 12 horas por cinco dias. A tetraciclina (vo) na dose de $500 \mathrm{mg}$ a cada oito horas, durante sete dias. A mefloquina em dose oral única de $15 \mathrm{mg} / \mathrm{kg}$, até a dose máxima de $1.000 \mathrm{mg}$.

Internação, observação e seguimento. Uma história clínica detalhada foi realizada no momento da admissão. O seguimento clínico se fez diariamente durante a internação e, posteriormente, nos dias 7, 14, 21 e 28 . A temperatura oral foi mensurada a cada seis horas, o termômetro permaneceu pelo menos três minutos na cavidade oral antes da leitura. $\mathrm{Na}$ admissão coletou-se uma amostra de sangue para a realização de hemograma e bioquímica sanguínea (transaminases, bilirrubinas, uréia, creatinina e glicemia). Estes exames foram repetidos nos dias 3 e 7 pós-tratamento. A contagem parasitária se realizou a cada 12 horas durante a internação e nos dias marcados para o seguimento clínico. Nenhuma droga antimalárica adicional foi usada nos pacientes durante os 28 dias de seguimento.

Remoção. Aqueles pacientes que apresentaram falha terapêutica (RII ou RIII)31, foram removidos do estudo. Toda recrudescência após sete dias foi tratada com dose única de mefloquina.
Critérios de avaliação. A eficácia foi avaliada pelo tempo de desaparecimento da febre (TDF), o tempo de clareamento parasitário (TCP) e a porcentagem de cura.

Avaliação estatística. Os dados foram analisados com o programa estatístico SAS (SAS Institute Inc., 1985). Todos os dados de distribuição normal foram comparados por análise de variância (ANOVA) segundo o teste de StudentNewman-Keuls (SNK). Os resultados em porcentagens foram analisados mediante teste de Mantel-Haenszel.

\section{RESULTADOS}

Características da população. As principais características da população encontram-se resumidas na Tabela 1 . Trinta e cinco (83,3\%) pacientes pertenciam ao sexo masculino e sete $(16,6 \%)$ ao feminino, com idades que variam de 8 a 64 anos (média 31,7; desvio padrão 12,9). A maioria dos pacientes procedia de Porto Velho $(76,2 \%)$ e a ocupação mais comum foi garimpeiro (23,8\%). Do total da amostra, 25 $(51,2 \%)$ pacientes moravam há mais de 10 anos em Rondônia; 39 (92,9\%) pacientes relataram haver tido pelo menos um episódio prévio e, somente $3(7,1 \%)$, eram primoinfectados. Uma alta porcentagem $(48,7 \%)$ de pacientes relatou ocorrência do último ataque de malária no mês prévio à internação. A ANOVA não demonstrou diferenças, estatisticamente significativas, quando comparadas às características populacionais dos grupos $(\mathrm{p}>0,05)$.

Tabela 1 - Características da população de acordo com grupos terapêuticos.

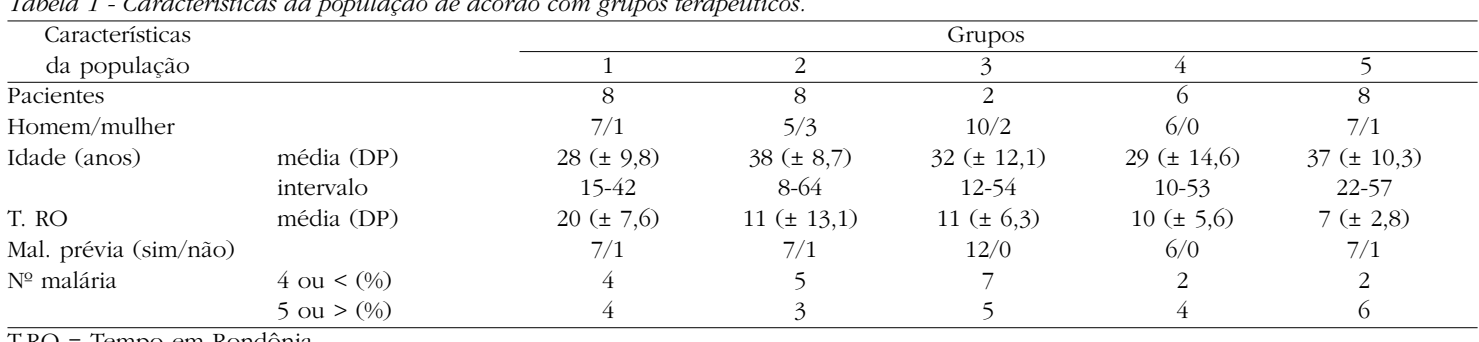

T.RO $=$ Tempo em Rondônia.

Características clinicas. O início dos sintomas precedeu em média, 4,3 dias antes do paciente procurar o atendimento médico. Os sintomas mais importantes foram febre e cefaléia, referidos por mais de $90 \%$ dos pacientes. Em relação aos sinais vitais no momento do ingresso (Tabela 2), a análise de variância não mostrou diferença significativa $(p>0,05)$ entre os grupos.
Exames laboratoriais de ingresso. Os resultados dos exames iniciais estão resumidos na Tabela 3. A análise estatística destes resultados não demonstrou diferença significativa entre os grupos $(\mathrm{p}>0,05)$.

Porcentagem de cura. Para a análise estatística, os pacientes foram agrupados pelo medicamento usado, sem importar a via de 
Salcedo JMV, Camargo LMA, Braga MFV, Maria PS, Macêdo VO. Avaliação da eficácia do artesunato associado à tetraciclina na terapêutica da malária falciparum. Revista da Sociedade Brasileira de Medicina Tropical 30:215-222, mai-jun, 1997.

administração. Dezeseis (38\%) pacientes tiveram acompanhamento inferior a 28 dias e foram classificados como sensíveis ou R I31. Vinte e seis (62\%) pacientes foram acompanhados por 28 ou mais dias. Um paciente de cada grupo apresentou R I tardia e um paciente do grupo do quinino, apresentou $\mathrm{R}$ III $\mathrm{e}$ foi removido do estudo. A porcentagem de cura, foi calculada com base nos 26 pacientes que completaram o seguimento de 28 dias. Para o artesunato foi $88,8 \%$, para a mefloquina $85,7 \%$ e para o quinino foi $81,8 \%$, sem diferença, estatisticamente significativa $(\mathrm{p}=0,6769)$.

Tempo de desaparecimento da febre (TDF). O grupo do artesunato apresentou desaparecimento da febre em 20,25 \pm 5,2 horas, o grupo da mefloquina em $32,44 \pm 17,67 \mathrm{e}$, o grupo do quinino, em 34,7 $\pm 17,3$ horas, sem diferença significativa entre eles $(\mathrm{p}=0,145)$.
Tempo de clareamento da parasitemia (TCP). O TCP para o grupo do artesunato foi de $37,33 \pm 11,52$ horas, para o grupo da mefloquina de $58,9 \pm 16,68$ horas e para o grupo do quinino de $65,25 \pm 17,44$ horas. De acordo com a via de administração, os resultados do TCP encontram-se resumidos na Tabela 4. A análise de variância, demonstra uma diferença, estatisticamente significativa $(\mathrm{p}=0,0016)$, entre o grupo de artesunato (oral/endovenoso) e o grupo do quinino (oral/endovenoso). O grupo da mefloquina não apresentou diferença significativa $(\mathrm{p}>0,05)$, quando comparado com os outros dois grupos. Não existe diferença em relação à via de administração (oral ou endovenoso) para estes medicamentos ( $\mathrm{p}>0,05)$.

Efeitos colaterais. Foram mínimos e autolimitados em todos os grupos. Todos os pacientes queixaram de cefaléia e mal estar

Tabela 2 - Sinais vitais no ingresso de acordo ao grupo terapêutico.

\begin{tabular}{|c|c|c|c|c|c|}
\hline \multirow{2}{*}{$\begin{array}{l}\text { Sinais } \\
\text { Vitais }\end{array}$} & \multicolumn{5}{|c|}{ Grupos } \\
\hline & 1 & 2 & 3 & 4 & 5 \\
\hline Temperatura (oC) & $37,5( \pm 0,6)$ & $38,2( \pm 0,7)$ & $37,8( \pm 0,9)$ & $38,5( \pm 0,7)$ & $37,8( \pm 1,1)$ \\
\hline Pulso (bat/min) & $85( \pm 6,3)$ & $95,2( \pm 9,2)$ & $88,5( \pm 7,5)$ & $87,3( \pm 9,8)$ & $88,3( \pm 6,8)$ \\
\hline TAS (mm/Hg) & $111( \pm 10,7)$ & $121( \pm 20,3)$ & $113( \pm 14,5)$ & $105( \pm 8)$ & $107( \pm 18)$ \\
\hline TAD $(\mathrm{mm} / \mathrm{Hg})$ & $71( \pm 8,6)$ & $75( \pm 16)$ & $71( \pm 8,7)$ & $65( \pm 10,5)$ & $67( \pm 7,6)$ \\
\hline
\end{tabular}

Medidas em média e desvio padrão; TAS = tensão arterial sistólica; TAD = tensão arterial diastólica

geral, sendo impossível diferenciar entre a sintomatologia da infecção e o efeito colateral. No grupo do artesunato, foram referidos diarréia $(12,5 \%)$, dor abdominal (12,5\%) e náuseas $(12,5 \%)$, os quais remitiram espontaneamente. No grupo da mefloquina, a diarréia foi referida em 25\% e a dor abdominal em 16,6\% dos pacientes, sendo também autolimitados. No grupo do quinino, foi referido dor abdominal em 37,5\% e diarréia em 12,5\% dos pacientes. A análise de variância dos valores laboratoriais pré e póstratamento (Tabela 5), usando o teste T de Student, não se demonstrou diferença estatisticamente significativa entre estes valores $(\mathrm{p}>0,05)$.

Tabela 3 - Características laboratoriais pré-tratamento por grupo terapêutico.

\begin{tabular}{|c|c|c|c|c|c|}
\hline \multirow[b]{2}{*}{ Exames laboratoriais } & \multicolumn{4}{|c|}{ Grupos } & \multirow[b]{2}{*}{5} \\
\hline & 1 & 2 & 3 & 4 & \\
\hline \multicolumn{6}{|l|}{ Parasitemia (por ml) } \\
\hline média & 29.405 & 32.810 & 71.612 & 4.800 & 52.371 \\
\hline desvio padão & 31.732 & 56.825 & 57.550 & 2.774 & 88.424 \\
\hline Hematócrito (\%) & $8( \pm 5,8)$ & $42( \pm 7,8)$ & $36( \pm 6,0)$ & $37( \pm 6,7)$ & $37( \pm 7,6)$ \\
\hline \multicolumn{6}{|l|}{ Leucócitos (por ml) } \\
\hline média & 6.125 & 5.300 & 6.420 & 4.867 & 5.800 \\
\hline Segmentados (\%) & $54( \pm 10,3)$ & $62( \pm 17,3)$ & $63( \pm 8,4)$ & $55( \pm 14,4)$ & $60( \pm 8,9)$ \\
\hline Bilirrubina direta $(\mathrm{mg} \%)$ & $0,46( \pm 0,47)$ & $0,59( \pm 0,23)$ & $0,69( \pm 0,59)$ & $0,68( \pm 0,56)$ & $0,53( \pm 0,37)$ \\
\hline Bilirrubina indireta $(\mathrm{mg} \%)$ & $0,51( \pm 0,71)$ & $0,51( \pm 0,26)$ & $0,51( \pm 0,32)$ & $0,72( \pm 0,54)$ & $0,34( \pm 0,25)$ \\
\hline TGO (U/L) & $7( \pm 14,8)$ & $40( \pm 9,6)$ & $21( \pm 9,4)$ & $22( \pm 5,4)$ & $9,7( \pm 4,7)$ \\
\hline TGP (U/L) & $22( \pm 39)$ & $36( \pm 12,8)$ & $16( \pm 11)$ & $17( \pm 8,5)$ & $9( \pm 6,3)$ \\
\hline Uréia $(\mathrm{mg} \%)$ & $32,6( \pm 6,2)$ & $28( \pm 6,9)$ & $33,6( \pm 8,9)$ & $35,3( \pm 17)$ & $33,3( \pm 15)$ \\
\hline Creatinina $(\mathrm{mg} \%)$ & $0,84( \pm 0,26)$ & $0,77( \pm 0,24)$ & $0,99( \pm 0,20)$ & $0,87( \pm 0,24)$ & $1,05( \pm 0,40)$ \\
\hline
\end{tabular}


Salcedo JMV, Camargo LMA, Braga MFV, Maria PS, Macêdo VO. Avaliação da eficácia do artesunato associado à tetraciclina na terapêutica da malária falciparum. Revista da Sociedade Brasileira de Medicina Tropical 30:215-222, mai-jun, 1997.

Tabela 4 - Clareamento da parasitemia de acordo com os grupos terapêuticos.

\begin{tabular}{lcccc}
\hline & \multicolumn{3}{c}{ Grupos } \\
\cline { 2 - 5 } & 1 & 2 & 3 & 4 \\
\hline TCP (horas) & 39,4 & 35,5 & 58,7 & 72,6 \\
Média & 15,3 & 7,56 & 16,4 & 9,1 \\
Desvio padrão & $21-65$ & $23-47$ & $37-83$ & $60-82$ \\
Intervalo & & $35-81$ \\
\hline Grupos: 1 = artesunato oral + tetraciclina; 2 = artesunato endovenoso + tetraciclina; 3 = mefloquina oral; 4 = quinino endovenoso + \\
tetraciclina; 5 = quinino oral + tetraciclina.
\end{tabular}

Tabela 5 - Exames laboratoriais pré e pós tratamento por grupo terapêutico.

\begin{tabular}{|c|c|c|c|c|c|c|}
\hline \multirow{3}{*}{$\begin{array}{l}\text { Exames } \\
\text { laboratoriais }\end{array}$} & \multicolumn{6}{|c|}{ Medicamento } \\
\hline & \multicolumn{2}{|c|}{ artesunato } & \multicolumn{2}{|c|}{ mefloquina } & \multicolumn{2}{|c|}{ quinino } \\
\hline & pré & pós & pré & pós & pré & pós \\
\hline Hematócrito & $39,6( \pm 7,4)$ & $35,7( \pm 4,2)$ & $36,4( \pm 6,0)$ & $36,9( \pm 4,5)$ & $37,4( \pm 7,2)$ & $33,6( \pm 5,3)$ \\
\hline Leucócitos (/ml) & 5.623 & 6.214 & 6.420 & 7.085 & 5.430 & 6.029 \\
\hline Segmentados (\%) & (15) & $(11,6)$ & $(8,4)$ & $(5,6)$ & $(11,7)$ & $(12,6)$ \\
\hline Bilirrubina indireta $(\mathrm{mg} \%)$ & $0,6( \pm 0,3)$ & $0,7( \pm 0,6)$ & $0,7( \pm 0,6)$ & $0,8( \pm 1,0)$ & $0,6( \pm 0,5)$ & $0,6( \pm 0,4)$ \\
\hline Bilirrubina direta $(\mathrm{mg} \%)$ & $0,6( \pm 0,6)$ & $0,6( \pm 0,6)$ & $0,5( \pm 0,3)$ & $0,3( \pm 0,3)$ & $0,6( \pm 0,5)$ & $0,5( \pm 0,5)$ \\
\hline TGO (UI/L) & $29,7( \pm 19,1)$ & $24,6( \pm 13,0)$ & $20,9( \pm 9,4)$ & $14,5( \pm 8,3)$ & $14,7( \pm 7,8)$ & $13,5( \pm 7,4)$ \\
\hline TGP (UI/L) & $31,6( \pm 35,7)$ & $34,0( \pm 16,2)$ & $16,5( \pm 11,1)$ & $15,8( \pm 10,7)$ & $12,6( \pm 8,2)$ & $12,5( \pm 10)$ \\
\hline Uréia (mg\%) & $30,6( \pm 7,2)$ & $21,8( \pm 5,8)$ & $33,6( \pm 8,9)$ & $22,9( \pm 7,7)$ & $34,1( \pm 15,4)$ & $21( \pm 11)$ \\
\hline Creatinina $(\mathrm{mg} \%)$ & $0,8( \pm 0,3)$ & $0,8( \pm 0,2)$ & $1,0( \pm 0,2)$ & $0,8( \pm 0,1)$ & $0,9( \pm 0,3)$ & $0,8( \pm 0,2)$ \\
\hline
\end{tabular}

Média e desvio padrão

Grupos: 1 = artesunato oral + tetraciclina; $2=$ artesunato endovenoso + tetraciclina; $3=$ mefloquina oral; $4=$ quinino endovenoso + tetraciclina; 5 = quinino oral + tetraciclina.

\section{DISCUSSÃO}

Nos últimos anos, muitos dos medicamentos antimaláricos têm diminuído sua efetividade, criando desta forma uma ameaça para o controle desta endemia2.

A artemisinina e seus derivados representam uma alternativa eficaz no tratamento da malária. Estes medicamentos têm clareamento parasitário rápido com poucos efeitos colaterais, contudo, sua porcentagem de cura é menor quando usados isoladamentes 13 , fato que motivou o uso de associações com antimaláricos de ação esquizonticida lenta.

Os sintomas referidos pelos pacientes, assim como os sinais vitais, os achados clínicos e os exames laboratoriais no momento de ingresso ao estudo, não diferiram daqueles descritos por outros autores em estudos terapêuticos similares16 18.

Dos 42 pacientes, 16 foram acompanhados menos de 28 dias e, 26 durante 28 ou mais dias, fato que reflete o alto índice de migração da população devido a problemas socioeconômicos e demográficos já descritos na região.

A porcentagem de cura do artesunato varia quando usado isolado ou em associação. Quando usado isoladamente, a porcentagem oscila de $42,9 \% 34$ até $92,5 \% 5$, dependendo da dose total e do tempo de tratamento; quando usado em associação esta varia de $95 \% 11$ a 100\%18.

No presente estudo, esta porcentagem $(88,8 \%)$ pode ser classificada como satisfatória segundo Rieckman 24 e resulta comparável com outros estudos 1118.

Peto e cols atentam para a relação que pode haver entre a alta porcentagem de recrudescência deste medicamento com os cursos curtos de tratamento ( 3 dias)22. Por este motivo, usamos um curso intermédio de artesunato ( 5 dias), associado à tetraciclina, na tentativa de alcançar uma porcentagem de cura similar à descrita na literatura. Atingiu-se este objetivo já que não encontramos diferença significativa entre outros estudos analisados e o nosso5 1118 .

É provável que a recrudescência tenha relação direita com o mecanismo de ação destes compostos e com a fase evolutiva do plasmódio, na qual atuam. O bloqueio da síntese de proteínas parece ser o alvo primário12, embora outros autores indiquem a geração de intermediários tóxicos de oxigênio, como mecanismo15. O alvo da ação destes medicamentos são as formas em 
Salcedo JMV, Camargo LMA, Braga MFV, Maria PS, Macêdo VO. Avaliação da eficácia do artesunato associado à tetraciclina na terapêutica da malária falciparum. Revista da Sociedade Brasileira de Medicina Tropical 30:215-222, mai-jun, 1997.

anéis maduras e trofozoítos jovens, sendo que as formas em anéis jovens e os esquizontes maduros são resistentes 6 .

Analisando em conjunto o descrito, com o fato destes medicamentos terem uma vida média curta $(0,45$ a 6 horas $)$ e, a prática de cursos de tratamento curtos (menos de cinco dias), é possível concluir que os anéis jovens e os esquizontes maduros estariam em capacidade de completar outro ciclo biológico, após terminado o tratamento antimalárico, determinando uma alta porcentagem de recaídas quando usados isoladamente em tratamentos de curta duração.

O tempo de desaparecimento da febre (TDF), não mostrou diferença estatística entre o grupo do artesunato $(20,25 \pm 5,2$ horas $)$, da mefloquina e do quinino $(32,44 \pm 17,67$ e $34,7 \pm 17,3$ horas, respectivamente). Estes resultados podem não estar mostrando significado estatístico devido ao pequeno número de pacientes incluídos no estudo. Bunnag e cols5, encontraram que o TDF variou de 20 a 29 horas, quando era usado artesunato oral, durante sete e cinco dias, respectivamente, resultado comparável com o descrito por nós. Ao que parece, a combinação com outros antimaláricos não altera este indicador.

O tempo de clareamento da parasitemia30 (TCP) foi $37,33 \pm 11,52$ horas para $\mathrm{o}$ artesunato, $58,9 \pm 16,68$ horas para a mefloquina e $65,25 \pm$ 17,44 horas para o quinino. Estes achados mostram que existe diferença significativa entre o grupo do artesunato e do quinino, porém, a mefloquina não difere de nenhum desses dois grupos. É importante esclarecer, que o TCP não expressa fielmente a atividade intrínseca antimalárica do medicamento, nem a segurança deste em impedir o aparecimento de complicações. A severidade da doença parece estar mais em relação ao número de parasitas sequestrados 29 e o grau de maturidade destes 26 , do que com seu número total. Por isto é impossível estabelecer relação direta entre a rapidez no clareamento da parasitemia e a melhora nos índices de sobrevida.

O TCP destes medicamentos não varia com o quadro clínico da malária, a via de administração ou o tempo de duração do tratamento. Hien e cols14, trabalhando com malária grave, descreveram um TCP semelhante para o artesunato endovenoso e oral. Comparado este tempo, com o descrito por Karbwang e cols16, para o artemeter oral, não se encontra diferença significativa. Bunnag e cols5, descreveram o mesmo TCP usando artesunato oral isoladamente, por cinco ou sete dias.

Por outro lado, este indicador varia de acordo com a presença ou não de sinergismo nas associações. Naing e cols21, relataram o maior TCP destes compostos até o momento descrito, quando associados à sulfadoxina/pirimetamina (106,7 \pm 48,7 horas). Já a combinação com mefloquina 28 , a qual tem demonstrado sinergismo in vitro, não altera significativamente este tempo (45,2 $\pm 16,6$ horas).

No Brasil, a experiência com estes compostos aumentou nos últimos anos. Boulos 3 , usando artemeter e artesunato isoladamente, encontrou um TCP de 52,7 e 57,5 horas, respectivamente. Em nosso estudo, o TCP da associação artesunato e tetraciclina, não difere dos achados na literatura e, pode indicar efeito aditivo in vivo destes compostos.

Não houve efeitos adversos importantes com nenhum dos esquemas usados. Cefaléia e tonturas foram as queixas mais comuns, possivelmente relacionadas mais com anemia e debilidade. Dor abdominal e a diarréia foram referidas em todos os grupos (variando de $12,5 \%$ a $37,5 \%$ ) sendo esta porcentagem, maior do que a encontrada na literatura (2\%18 a 9\%16). É provável que a tetraciclina ou a presença de patologias associadas (helmintíases ou protozooses) possam explicar em parte, este fato. Nenhuma alteração psicológica ou psiquiátrica foi observada no grupo da mefloquina.

Em relação aos exames laboratoriais, a queda nas cifras do hematócrito foi comum para os grupos e parece estar mais relacionada com a própria malária do que com os medicamentos. Bunnag e cols 4 relatam diminuição nas cifras de leucócitos segmentados, também encontrada neste estudo, porém, sem significância estatística. Nenhum dos resultados dos exames laboratoriais variou com o tratamento.

A primeira e última vez que um produto natural foi aceito para tratar malária, foi no século XVI. A artemisinina e seus derivados abriram novos horizontes na terapêutica da malária, sendo provável que estejamos assistindo uma nova era no controle desta endemia.

São necessários outros estudos para avaliar se existe associação entre rapidez no clareamento da parasitemia e melhora nos indices de sobrevida. 
Salcedo JMV, Camargo LMA, Braga MFV, Maria PS, Macêdo VO. Avaliação da eficácia do artesunato associado à tetraciclina na terapêutica da malária falciparum. Revista da Sociedade Brasileira de Medicina Tropical 30:215-222, mai-jun, 1997.

\section{SUMMARY}

A controlled clinical therapeutic study in bospitalized patients compared artesunate with quinine and mefloquine in patients with uncomplicated falciparum malaria. Forty two patients entered the trial and the follow up was for 28 days with thick blood film taken every 12 bours untill became negative. Laboratory examinations included haematological and biochemical tests before and after treatment. Patients had a mean parasitaemia of 42.568 per microlitre. Twenty six patients completed 28 days of follow up but 16 did not fulfil this protocol. One in each of the therapeutic groups showed delayed $R$ I resistance. A further patient in the quinine group showed $R$ III resistance. The cure rate was $88.8 \%$ for artesunate, $85.7 \%$ for mefloquine and $81.8 \%$ for quinine; no significant difference was found, the same occurring with the clearance of fever. The artesunate group had a quicker parasitaemia clearance time (37.3 \pm 11.5 hours) when compared with quinine (65.2 \pm 17.4$)$ showing a significant diference $(p=0.0016)$. Parasite clearance with mefloquine, was intermediate (58.9 \pm 16.6 ours) between the artesunate and quinine. No important side effects were observed with any of the therapeutic regimens and no deaths registered.

Key-words: Malaria. Artemisinin. Artesunate Clinic assay.

\section{AGRADECIMENTOS}

Aos Profs. Philip D. Marsden e João Barberino Santos pelas críticas e sugestões. A toda equipe do CEMETRON; Ao Dr. Aluízio Prata e à Dra ${ }^{\mathrm{a}}$ Otávia Caballero do Hospital Escola da Faculdade de Medicina do Triângulo Mineiro; ao Dr. Francisco R. dos Santos da UNIMED/Rondônia.

\section{REFERÊNCIAS BIBLIOGRÁFICAS}

1. Anonymus. Chinese Cooperative Research Group on Qinghaosu and Derivatives as Antimalarials (English abstract). Journal of Traditional Chinese Medicine 2: 1-25, 1982.

2. Björkman A, Phillips-Howard PA.The epidemiology of drug resistance of malaria. Transaction of the Royal Society of Tropical Medicine and Hygiene 84: 177-180, 1990.

3. Boulos M. Resistência do P. falciparum aos derivados da artemisinina. In: Resumenes I
Congreso Latinoamericano de Medicina Tropical. Guayaquil, p.73, 1993.

4. Bunnag D, Viravan C, Looareesuwan S, Karbwang J, Harinasuta T. Double blind randomised clinical trial of two different regimens of oral artesunate in falciparum malaria. Southeast Asian Journal of Tropical Medicine and Public Health 22: 534-538, 1991.

5. Bunnag D, Viravan C, Looreesuwan S, Karbwang J, Harinasuta T. Double blind randomised clinical trial of oral artesunate at once or twice daily dose in falciparum malaria. Southeast Asian Journal of Tropical Medicine and Public Health 22: 539-543, 1991.

6. Caillard V, Beaute-Lafitte A, Chabaud AG, Landau I. Plasmodium vinckei petteri: identification of the stages sensitive to arteether. Experimental Parasitology 75: 449-456, 1992.

7. Chawira NA, Warhurst DC. The effect of artemisinin combined with standard antimalarial against chloroquine-sensitive and chloroquineresistant strains of $P$. falciparum in vitro. Journal of Tropical Medicine and Hygiene 90:1-5, 1987.

8. Colwell EJ, Hickman RL, Intraprasert R, Tirabutana C. Minocycline and tetracycline treatment of acute falciparum malaria in Thailand. American Journal of Tropical Medicine and Hygiene 21:144148, 1972.

9. Dobenstyn FB, Phintuyothin P, Noeypatimanondh $S$, Teerakiarkamjorn S. Single dose therapy of falciparum malaria with mefloquine and pyrimethamine-sulfadoxine. Bulletin of World Health Organization 57:275-278, 1979.

10. Fontanet AL, Johnston BD, Walker AM, Rooney W, Thimasarn K, Sturchler D, Macdonald M, Hours M, Wirth DF. High prevalence of mefloquineresistant falciparum malaria in Eastern Thailand. Bulletin of World Health Organization 71:377383, 1993.

11. Fontes CJF, Pereira JCC, Grzesiuk AK. Artesunato oral associado a tetraciclina no tratamento da malária causada por P. falciparum. In: Resumos XXIX Congresso da Sociedade Brasileira de Medicina Tropical. Fortaleza, p. 247, 1993.

12. Gu HM, Warhurst DC, Peters W. Rapid action of qinghaosu and related drugs on the incorporation of $(3 \mathrm{H})$ isoleucine by $P$. falciparum in vitro. Biochemical Pharmacology 32: 2463-2466, 1983.

13. Guoqio Li, Arnold K, Xingbo Guo, Hwaxing Jian, Linchun Fu. Randomised comparative study of mefloquine, qinghaosu and pyrimethamine- 
Salcedo JMV, Camargo LMA, Braga MFV, Maria PS, Macêdo VO. Avaliação da eficácia do artesunato associado à tetraciclina na terapêutica da malária falciparum. Revista da Sociedade Brasileira de Medicina Tropical 30:215-222, mai-jun, 1997.

sulfadoxine in patients with falciparum malaria. Lancet II:1360-1361, 1984.

14. Hien TT, Phu NH, Mai NT, Chau TT, Trang TT, Loc PP, Cuong BM, Dung NT, Vinh H, Waller DJ. An open randomised comparison of intravenous and intramuscular artesunate in severe falciparum malaria. Tranansactions of the Royal Society of Tropical Medicine and Hygiene 86:584-585, 1992.

15. Howells RE. The modes of action of some antiprotozoal drugs. Parasitology 90:687-703, 1985.

16. Karbwang J, Bangchang KN,Thanavibul A, Bunnag $\mathrm{D}$, Chongsuphajaisiddhi T, Harinasuta T. Comparison of oral artemether and mefloquine in acute uncomplicated falciparum malaria. Lancet 340:1245-1248, 1992.

17. Klayman DL. Qinghaosu (Artemisinin): an antimalarial from China. Science 228: 1040-1047, 1985.

18. Looreesuwam S, Kyle DE,Viravan C,Vanijanonta S, Wilairatana P, Charoenlarp P, Canfield CJ, Webster HK. Treatment of patients with recrudescent falciparum malaria with sequential combinations of artesunate and mefloquine. The American Journal of Tropical Medicine and Hygiene 47:794799, 1992.

19. Looreesuwam S, Viravan C, Vanijanonta $S$, Wilairatana $\mathrm{P}$, Suntharasamai $\mathrm{P}$, Charoenlarp $\mathrm{P}$, Arnold K, Kyle DE, Canfield CJ, Webster HK. A randomized trial of artesunate and mefloquine alone and in sequence for acute uncomplicated falciparum malaria. Lancet 339:821-824, 1992.

20. Ministério da Saúde. Gerência Técnica da Malária, FNS. Situação da malária no Brasil, tendências e perspectivas, 1992.

21. Naing T, Win H, Nwe YY, Pe Than Myint, Tin Shwe. The combined use of artemether, sulfadoxine and pyrimethamine in the treatment of uncomplicated falciparum malaria. Transaction of the Royal Society of Tropical Medicine and Hygiene 82:530$531,1988$.

22. Peto TEA. Qinghaosu, mefloquine and pyrimethamine-sulfadoxine in falciparum malaria. Lancet I:216, 1985.

23. Reyes S, Passos ADC, Osanai CH. Resistência in vivo do $P$. falciparum às 4-aminoquinoleínas e à associação sulfadoxina-pirimetamina. I. Estudo de Porto Velho, Rondônia, 1983. Revista da Sociedade Brasileira de Medicina Tropical 18:175-181, 1985.
24. Rieckmann KH. Monitoring the response of malaria infection to treatment. Bulletin of World Health Organization 68: 759-763, 1990.

25. Sampaio LS, Silva EB, Sampaio MZM, Queiroz Silva VMF de, Carvalho EB da S,Alecrim MGC.Tratamento da malária por $P$. falciparum com artemisinina oral. In: Resumos do XXIX Congresso da Sociedade Brasileira de Medicina Tropical. Fortaleza p. 127, 1993.

26. Silamut K, White NJ. Relation of the stage of parasite development in the peripheral blood to prognosis in severe falciparum malaria.Transaction of the Royal Society of Tropical Medicine and Hygiene 87:436-443, 1993.

27. Ter Kuile F, White NJ, Holloway P, Pasvol G, Krishna S. Plasmodium falciparum: in vitro studies of the pharmacodynamic propierties of drugs used for the treatment of severe malaria. Experimental Parasitology 76:85-95, 1993.

28. Tin Shwe, Pe Than Myint, Win Myint, Ye Htut, Lin Soe, Min Thwe. Clinical studies on treatment of cerebral malaria with artemether and mefloquine. Transaction of the Royal Society of Tropical Medicine and Hygiene 83:489-490, 1989.

29. White NJ, Ho M. Pathophysiology of malaria. Advances in Parasitology 31:84-175, 1992.

30. White NJ, Krishna S. Treatment of malaria: some considerations and limitations of the current methods of assessment. Transaction of the Royal Society of Tropical Medicine and Hygiene 83:767$777,1989$.

31. World Health Organization. Chemotherapy of malaria and resistance to antimalarials. World Health Organization, Geneve,T.R.S. NO 529, 1973.

32. World Health Organization. Basic laboratory methods in medical parasitology. Geneve, World Health Organization p. 80-88, 1991.

33. World Health Organization. World Malaria Situation 1990. World Health Statistics Quarterly 45: 257-266, 1992.

34. World Health Organization. The role of Artemisininand its derivatives in the current treatment of malaria (1994-1995). Geneve, World Health Organization, 1994.

35. Young MD, Moore DV. Chloroquine resistance in $P$. falciparum. The American Journal of Tropical Medicine and Hygiene 10:317, 1961. 